\title{
Probing Stellar Mass Assembly in the Virgo Cluster
}

\author{
Joel C. Roediger ${ }^{1}$, Stéphane Courteau ${ }^{1}$, \\ Michael McDonald ${ }^{2}$ \& Lauren A. MacArthur ${ }^{3}$ \\ ${ }^{1}$ Department of Physics, Engineering Physics \& Astronomy, Queen's University, \\ 99 University Avenue, Kingston, Ontario, K7L 3N6 Canada \\ email: jroediger@astro.queensu.ca; courteau@astro.queensu.ca \\ ${ }^{2}$ Department of Astronomy, University of Maryland, \\ College Park, MD, 20742-2421 U.S.A. \\ ${ }^{3}$ NRC Herzberg Institute of Astrophysics, \\ 5071 West Saanich Road, Victoria, B.C., V9E 2E7 Canada
}

\begin{abstract}
We have examined in detail both the resolved and integrated stellar content of a representative sample of galaxies in the Virgo cluster to constrain plausible formation/evolution scenarios for them. Systematic variations in age and metallicity gradients along the Hubble sequence are found such that the gradients become more negative for later types. Combined with correlations of the above stellar properties with both structure and environment, these data highlight the significance of merging in the assembly of massive spheroids and environmental phenomena (gas removal/interactions) in the evolution of disks and dwarf systems. Our data also provide useful perspectives on a number of important topics in the recent literature.
\end{abstract}

Keywords. clusters: individual (Virgo), galaxies: evolution, formation, general, stellar content

Stellar light encodes information on both the time elapsed since formation (star formation history, $\mathrm{SFH}$ ) and prior chemical evolution (CE) of the interstellar medium. Stellar populations (SPs) in nearby galaxies thus provide boundary conditions for inferences from galaxy formation/evolution simulations and studies at high- $z$ (e.g., REF). However, the most comprehensive study of local SPs yet (MacArthur et al. 2004) has only examined gas-rich systems. We summarize here results from our SP analysis of 285 systems in the Virgo cluster in order to assess SFHs and CE, and hence the evolution, of galaxies of all types.

For our study we use the database of resolved optical (gri) and near-infrared (NIR; $H$ ) photometry from McDonald et al. (in prep.). This sample contains all galaxy types, and so probes the full range in galaxian parameters. Correlations of SP properties with galaxy structure and environment are explored with non-parametric NIR structural quantities (concentration, $C_{28}$; apparent magnitude, $H$; effective surface brightness, $\mu_{e}$, and radius, $r_{e}$ ) and $D e f_{H I}$ values drawn from McDonald et al. (2009) and Gavazzi et al. (2005), respectively. We also use the environmental diagnostics of cluster-centric distance $\left(D_{M 87}\right)$ and surface density $(\Sigma)$.

To analyze the resolved and integrated SEDs of each galaxy, we use the simple stellar population models of Charlot \& Bruzual (in prep.) convolved with an exponential SFH of variable timescale. The best-fit combination of mean age, $\langle A\rangle$, and metallicity, $Z$, for each SED is determined via minimization of a simple $\chi^{2}$-estimator. The typical $\langle A\rangle$ and $Z$ profile for each principal galaxy type is shown in Fig. 1. We summarize major results from our analysis below. 


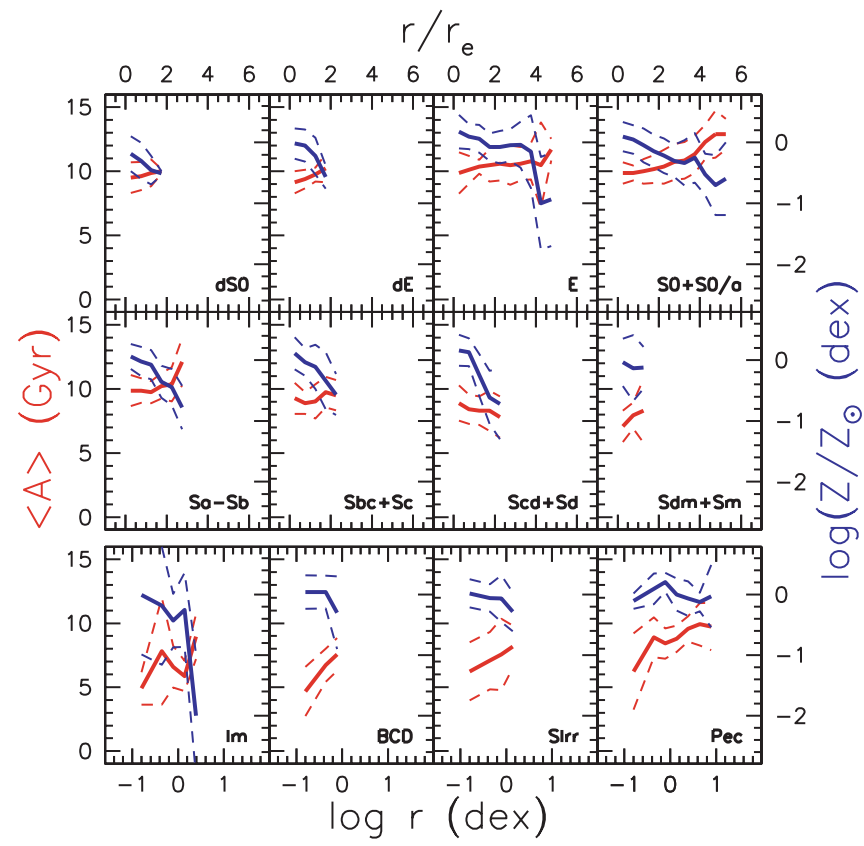

Figure 1. Median $\langle A\rangle$ (red) and $Z$ (blue) profiles for Virgo cluster galaxies. The profiles are separated into major morphological types/bins, as indicated in the lower-right corner of each window. The median trend and $1 \sigma$ (RMS) dispersion are represented by the solid and dashed lines, respectively. For irregular galaxies (Im, BCD, SIrr, Pec), the profiles are plotted against physical radii (in logarithmic units), as the typically low surface brightness of these systems translates to shallow profiles, relative to $r_{e}$.

General trends:

- $\langle A\rangle$ and $Z$ gradients systematically decrease toward later Hubble types (Fig. 1)

- Linear correlations of $\langle A\rangle$ and $Z$ with structural/environmental parameters are typically weak $(r \leqslant 0.4)$, for both local (at $\left.1 r_{e}\right)$ and global (i.e., integrated) measures

Early-type galaxies (dS0, dE, E, S0+S0/a):

- $\langle A\rangle$ and $Z$ variations within $2 r_{e}$ most consistent with dominance of gas-rich mergers

- SP-structure distributions indicate dwarfs and giants differ in their formation (e.g.,

$\langle A\rangle$ vs. $C_{28}$ ), and that the colour-magnitude relation results from metallicity variations Spiral galaxies $(\mathrm{Sa}-\mathrm{Sm})$ :

- $\langle A\rangle$ and $Z$ gradient combinations suggest unanimous merger origin for all bulges

- Density-dependent chemical enrichment is not confirmed (MacArthur et al. 2004)

- Strong $\langle A\rangle-D e f_{H I}$ correlations argue disk evolution governed by gas removal

Irregular galaxies (Im, BCD, SIrr, Pec):

- Similarly strong $\langle A\rangle-D e f_{H I}$ correlations suggest action of gas removal in Im+BCD's

- $\langle A\rangle-D_{M 87}-\Sigma$ trends for SIrr+Pec's argue for past interactions with cluster potential

\section{References}

Charlot, S. \& Bruzual, G. (in prep.)

Gavazzi, G., Boselli, A., van Driel, W., \& O'Neil, K. 2005, A\& A, 429, 439

MacArthur, L. A., Courteau, S., Bell, E., \& Holtzman, J. A. 2004, ApJS, 152, 175

McDonald, M., et al. (in prep.)

McDonald, M., et al. 2009, MNRAS, 394, 2022 